gratefully) lived through a clinical revolution which enabled us to pick up and lead normal lives.

At the time of writing I am, so far as I know, fit and privileged to have benefited from the dedication of those hundreds of tuberculosis specialists who literally nursed us through all the experimental treatments which began the medical revolution in the 1950 s and early 1960s. It is probably true that the public at large does not realise the vast change that has occurred. But the patients who survived will never forget it.

Neville Taylor

Wigmore, Kent

\section{A case of haemolytic-uraemic syndrome}

SIR,-The Clinicopathological Conference (21 July, p 179) presents and discusses an uncommon but catastrophic sequel to an otherwise normal pregnancy. I think $\mathrm{Dr}$ D Gwyn Williams's discussion is excellent, but I must draw attention to one or two points in it.

I agree that the data indicate the presence of a microangiopathic haemolytic anaemia; however, this condition is almost invariably associated with disseminated intravascular coagulation, and to state that defibrination was not evident because of a normal fibrinogen is erroneous. Plasmin fibrinogen concentration (we measure clottable protein rather than estimating the titre) is elevated in normal pregnancy by at least $100 \%$ at term and returns to normal non-pregnant values only very slowly. Thus the titre of $1 / 128$ represents a low value-in other words, hypofibrinogenaemia.

Pregnancy does indeed facilitate the generalised Schwartzman reactions-in animals at least-but I know of no evidence to implicate placental emboli, as these are found in a very large percentage of normal pregnancies. Dr J H H MacRae's comment vis-à-vis "obstetric mischief" is interesting if not provocative, and I wonder to what "powerful" labour-inducing drugs he refers.

I agree with $\mathrm{Dr}$ Williams that no drugs have been shown to be culpable. However, the synthetic oestrogens used for lactation suppression have been shown to enhance postpartum coagulability and to have an association with deep thrombosis in the puerperium. I know of no such relationship to microangiopathic haemolytic anaemia.

Finally, I agree that bilateral nephrectomy might have been considered at an early stage in the management of this tragic case.

Ashley T Coopland

Medical Center,

University of Colorado,

Denver, 80262 , USA

\section{Too few necropsies}

SIR,-It is not only a decline in necropsy numbers in hospital patients that is the problem: it is the standard of the necropsy, as $\mathrm{Dr}$ H A Fleming says (25 August, p 501). In a medical school the necropsy is thorough in every minute aspect; for the student pathologist has to learn, and even to research, the significance of all appearances. Away from such centres the pathologist can continue with his learning, teaching, and research; but it is the standard of his clinical-pathology con- ference now which has to be judged. I would define every necropsy as a conference of this kind-and one designed to check and countercheck the clinical significance of all findings. We must be aware, too, of the potential in every coroner's case.

Success depends on concerned clinicians and pathologists realising the limitations of their professed disciplines and thinking over the necropsy exhibits together, just before and during the actual cutting. When the macroscopic sectioning is finished all can discuss relevant matters on the spot; and from time to time histology, biochemistry, microbiology, and other subdisciplines may be called in to postpone decision when this is justified.

How many necropsies done at his request has Dr Fleming missed seeing and discussing in this way? I suggest that we encourage the habit of seeking to make such discussions happen, for then I am sure that fewer matters of relevance will remain undiscussed and undisclosed.

Pathology Laboratory,

York District Hospital

G A C SUMMers

\section{Abnormal cilia}

SIR,-Your recent leading article entitled "Abnormal cilia" (23 June, p 1663) ${ }^{1}$ refers to a topic of renewed interest in pathology: the possibility of a relationship between structural abnormalities of the cilia and impaired mucociliary functions.

Unfortunately, however, the article contains a number of errors. The main conclusion of the article is that "impaired mucociliary clearance, then, seems to be associated with structural abnormalities of the cilia." This is not necessarily so. In many-perhaps mostcases of impaired mucociliary clearance the entire ciliated epithelium is altered. A squamous metaplasia develops but there are no changes in the cilia as such. ${ }^{1}$ In the case of cystic fibrosis the mucociliary functions are impaired, but the cilia have been shown to have a perfectly normal structure. ${ }^{2}$ The explanation is simple. Mucociliary clearance is performed by a system with at least two components, and an error may reside in the mucus production or in the mucus properties rather than in the cilia.

It would be true, however, to say that structural abnormalities in the cilia will cause a defective or even absent mucocilary clearance. In my opinion there are several forms of inborn ciliary defects which all give rise to what has been called the immotile-cilia syndrome. ${ }^{34}$ These forms are characterised by deficiencies in either the outer dynein arms, the inner dynein arms, both the outer and inner dynein arms, the spoke heads and central sheath components, or some other essential component. ${ }^{5}$ There are also many other forms of inborn or acquired errors in the ciliary structure. When only some of the cilia are affected, as is the case with the clumping of cilia into what has been called compound cilia, then the influence on the mucociliary clearance may be negligable. It is recommended that cilia are examined in their living state for their motility, and that the electronmicroscopical sections are examined with respect to one characteristic of the cilia in the immotile-cilia syndrome-the loss of the normal, parallel orientation of the cilia.
It is to be noted that the cilia have nine microtubular doublets in a circle around the central two microtubules, and that these doublets (which are unlikely to be identical) have two dynein arms on their one component, the subfibre called the A-tubule. The description given in your leading article is quite confused.

In the last paragraph you say that further electron-microscopical studies of patients with chronic bronchitis are recommended, but that the rotational imaging technique for contrast enhancement has to be used. I cannot agree with the second point. With the rotational technique ${ }^{6}$ it is possible to get nearly any kind of image one wants to obtain. ${ }^{78}$ It is a technique which must be used with great caution, if at all. There is no substitute for taking electron micrographs that are good enough to make the individual structural components of the cilia-spokes or dynein arms-visible.

BJöRn Afzelius

Wenner-Gren Institute,

University of Stockholm

Stockholm, Sweden

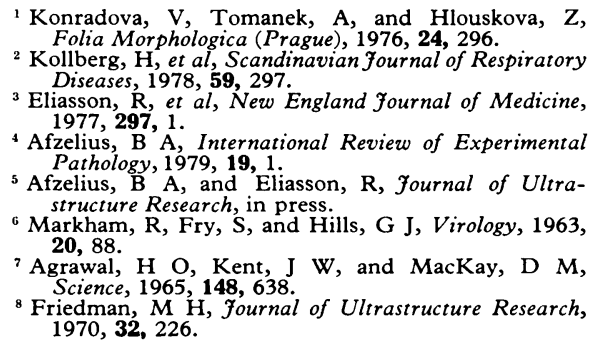

Umbilical hernia and abdominal cramp in swimmers

SIR,-I was most interested in the letter from Mr F D Skidmore (25 August, p 494) relating to umbilical hernia in swimmers. He inquires whether abdominal cramp in swimmers does in fact occur.

During a course for potential internationals some years ago a boy of 17 years, himself an international sprinter, ceased swimming, made for the pool side, and pulled himself out by the use of his arms. The whole of the abdominal musculature was in spasm. The spine was extended and hot towels were placed on the abdominal wall. After a while the spasm abated and he was able to have a hot shower and get dressed. There were no aftereffects; in fact, he trained at a session later in the day. Inquiry revealed that he had been in the gymnasium performing exercises with the medicine ball prior to training in the pool and his cramp was possibly the direct result of this activity.

$\mathrm{Mr}$ Skidmore's observation has brought to our notice the value of the examination of young athletes before embarking on training programmes, and the possible danger that could arise to those swimming alone.

Noel Bleasdale Honorary Medical Adviser

Hoylake, Cheshire Amateur Swimming Association

SIR,-It must be doubtful whether swimming training can cause umbilical hernia and equally doubtful whether a "small hernia" can cause gross abdominal muscle spasm and rapid splinted respiration, as suggested by $\mathrm{Mr}$ F D Skidmore (25 August, p 494). Even if $\mathrm{Mr}$ Skidmore is correct in linking umbilical 